\title{
Comparative genomic analysis of 142 bacteriophages infecting Salmonella enterica subsp. enterica
}

Ruimin Gao ${ }^{1,2^{*}}$, Sohail Naushad ${ }^{1}$, Sylvain Moineau ${ }^{3,4,5}$, Roger Levesque ${ }^{6}$, Lawrence Goodridge ${ }^{7}$ and Dele Ogunremi ${ }^{1 *}$ (D)

\begin{abstract}
Background: Bacteriophages are bacterial parasites and are considered the most abundant and diverse biological entities on the planet. Previously we identified 154 prophages from 151 serovars of Salmonella enterica subsp. enterica. A detailed analysis of Salmonella prophage genomics is required given the influence of phages on their bacterial hosts and should provide a broader understanding of Salmonella biology and virulence and contribute to the practical applications of phages as vectors and antibacterial agents.

Results: Here we provide a comparative analysis of the full genome sequences of 142 prophages of Salmonella enterica subsp. enterica which is the full complement of the prophages that could be retrieved from public databases. We discovered extensive variation in genome sizes (ranging from 6.4 to $358.7 \mathrm{~kb}$ ) and guanine plus cytosine (GC) content (ranging from 35.5 to 65.4\%) and observed a linear correlation between the genome size and the number of open reading frames (ORFs). We used three approaches to compare the phage genomes. The NUCmer/MUMmer genome alignment tool was used to evaluate linkages and correlations based on nucleotide identity between genomes. Multiple sequence alignment was performed to calculate genome average nucleotide identity using the Kalgin program. Finally, genome synteny was explored using dot plot analysis. We found that 90 phage genome sequences grouped into 17 distinct clusters while the remaining 52 genomes showed no close relationships with the other phage genomes and are identified as singletons. We generated genome maps using nucleotide and amino acid sequences which allowed protein-coding genes to be sorted into phamilies (phams) using the Phamerator software. Out of 5796 total assigned phamilies, one phamily was observed to be dominant and was found in 49 prophages, or $34.5 \%$ of the 142 phages in our collection. A majority of the phamilies, 4330 out of 5796 (74.7\%), occurred in just one prophage underscoring the high degree of diversity among Salmonella bacteriophages.

* Correspondence: ruimin.gao@canada.ca; dele.ogunremi@canada.ca ${ }^{1}$ Ottawa Laboratory Fallowfield, Canadian Food Inspection Agency, Ottawa, Ontario, Canada

Full list of author information is available at the end of the article

(c) The Author(s). 2020 Open Access This article is licensed under a Creative Commons Attribution 4.0 International License, which permits use, sharing, adaptation, distribution and reproduction in any medium or format, as long as you give appropriate credit to the original author(s) and the source, provide a link to the Creative Commons licence, and indicate if changes were made. The images or other third party material in this article are included in the article's Creative Commons licence, unless indicated otherwise in a credit line to the material. If material is not included in the article's Creative Commons licence and your intended use is not permitted by statutory regulation or exceeds the permitted use, you will need to obtain permission directly from the copyright holder. To view a copy of this licence, visit http://creativecommons.org/licenses/by/4.0/ The Creative Commons Public Domain Dedication waiver (http://creativecommons.org/publicdomain/zero/1.0/) applies to the data made available in this article, unless otherwise stated in a credit line to the data. 


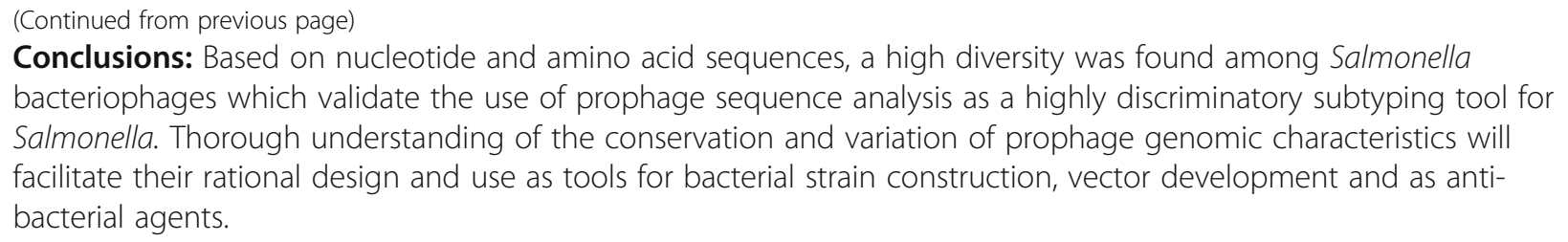

Keywords: Comparative genomics, Bacteriophage, Nucleotide identity, Salmonella enterica, Phamerator, Prophage sequence typing, Phage clusters

\section{Background}

The Gram-negative bacterial genus Salmonella belongs to the family Enterobacteriaceae, order Enterobacteriales, class Gammaproteobacteria and phylum Proteobacteria. Salmonella cells have a length of 2 to $5 \mu \mathrm{m}$ and a diameter ranging from 0.7 to $1.5 \mu \mathrm{m}$, as well as being predominantly motile due to peritrichous flagella [1]. The genus consists of two species, namely Salmonella enterica and S. bongori. The former can be further divided into six subspecies which corresponds to known serotypes (depicted with Roman numerals): enterica (I), salamae (II), arizonae (IIIa), diarizonae (IIIb), houtenae (IV) and indica (VI) [2]. The serotype V is now considered a separate species and designated $S$. bongori. Based on the presence of somatic $\mathrm{O}$ (lipopolysaccharide) and flagellar $\mathrm{H}$ antigens (Kauffman-White classification), the above six $S$. enterica subspecies are divided into over 2600 serovars [3] but fewer than 100 serovars have been associated with human illnesses [4]. Salmonella enterica subpecies enterica is typically categorized into typhoidal and non-typhoidal Salmonella as a result of symptoms presenting in infected humans. Non-typhoidal Salmonella, which is made up of a large number of the serovars, can be transmitted from animals to humans and between humans, often via vehicles such as foods, and they usually invade only the gastrointestinal tract leading to symptoms that resolve even in the absence of antibacterial therapy [5]. In contrast, typhoidal Salmonella serovars such as Typhi, Paratyphi A and Paratyphic C, are transferred from human to human and can cause severe infections requiring antibiotic treatment [6]. Wide spread resistance against antibiotics has prompted a renewed surge of interest in bacteriophages which are viruses capable of infecting and sometimes killing bacteria, as safe and effective therapy alternatives [7].

Bacteriophages, sometimes simply referred to as phages, are considered the most abundant biological entities on the planet [8]. These bacterial viruses can undergo two life cycles: lysis or lysogeny. A bacteriophage capable of only lytic growth is described as virulent. In contrast, temperate bacteriophage refers to the ability of some phages to display a lysogenic cycle and instead of killing the host bacterium becomes integrated into the chromosome. A bacterium that contains a set of phage genes representing an intact prophage is called a lysogen, while the integrated viral DNA is called a prophage. Most temperate phages form lysogens by integration at a unique attachment site in the host chromosome $[9,10]$. The integration process has been described as a biological arms race between the infecting virus and the host bacterium [11]. There is an array of host defense mechanisms that are stacked against the virus which in turn increasingly acquires and displays a counter-offensive to thwart and evade the anti-viral mechanisms resulting in integration into the host genome [11-13].

Tailed phages which belong to the Order Caudovirales are the most abundant group of viruses infecting bacteria and are also the most prevalent in the human gut. They are easily recognized under an electron microscope by their polyhedral capsids and tubular tails [14]. The order Caudovirales is made up of five families, namely: (1) Myoviridae (contractile tails, long and relatively thick), (2) Siphoviridae (long noncontractile tails), (3) Podoviridae (short noncontractile tails) [14, 4) Ackermannviridae (contractile tails) and (5) Herelleviridae - spouna-like (contractile tails, long and relatively thick) [15]. Bacteriophages were first described by Frederick Twort in 1915 and Felix d'Herelle in 1917 [16], and studies into their relationship with Salmonella enterica serovar Typhimurium led to the description of "symbiotic bacteriophages" by Boyd [17]. We recently analyzed the bacteriophages present in 1760 genomes of Salmonella strains present in a research database (https:// salfos.ibis.ulaval.ca/) and apart from three strains devoid of any prophage, the genomes had 1-15 prophages with an average of 5 prophages per isolate [18]. Previous analyses of Salmonella phages have led to their classification into five groups (P27-like, P2-like, lambdoid, P22-like, and T7-like) and three outliers ( $\varepsilon 15, \mathrm{KS} 7$, and Felix O1) [10]. Apart from the primary role of phage gene products to ensure that these viruses can infect bacteria, survive and reproduce in their hosts, phage genes have been shown to code for virulence factors, toxin, and antimicrobial resistance genes. The presence of these genes appears to contribute in a 
substantial manner to the evolution of the bacterial host [18-20]. Studies of prophage biology have practical significance in choice of phages as antibacterial agents, in bacterial strain construction and typing for epidemiological purposes [21, 22].

The advent of whole genome sequencing has greatly facilitated the detection and characterization of phages and prophages in bacterial hosts and the ability to evaluate their impacts on the host. Evolutionary analysis of phage genes open reading frames (ORF) families based on sequence analysis of a large number of phage genomes in the GenBank (about 13,703 phage genomes were present as of June 2019) (http://millardlab.org/bioinformatics/bacteriophagegenomes/phage-genomes-june-2019/) has provided insights into the impact on the evolution of both the virus and host [23]. Whole-genome comparative analysis has been successfully applied to study phages present or infecting several bacterial genera including Mycobacteria [24], Staphylococcus [25], Bacillus [26], Gordonia [27], Pseudomonas [23] and as well as the Enterobacteriaceae family [28]. Phage genomes are commonly grouped into clusters, but outlier phages lacking strong nucleotide identity relationships with other clustered genome are often designed as 'singletons' [27]. To classify phage genomes into clusters and subclusters, there are several commonly used tools/approaches. The dot plot program Genome Pair Rapid Dotter (Gepard) [29] can reveal very substantial synteny among genomes. Typically, the dot plot can recognize similarities spanning more than half of the genome lengths [24]. The average nucleotide identity (ANI) are determined using tools such as Kalign [30] and MUMmer [31] using genomes alignment and comparison. Genome map and gene content analyses can be performed using Phamerator, which assorts proteincoding genes into Phamilies (Phams) and generate a database of gene relationships [32, 33].

Using PHASTER (PHAge Search Tool Enhanced Release) [34, 35], we previously demonstrated the presence of 154 different prophages in 1760 S. enterica genomes which covered 151 Salmonella serovars [18]. We also previously showed that some prophage sequences were conserved among strains belonging to the same serovars and that the prophage repertories provided an additional marker for differentiating $S$. enterica subtypes during foodborne outbreaks [18]. Here, a more detailed characterization of these Salmonella phage genomes was carried out to generate knowledge on their biological variation and evolution and thereby provide insights into the role of phages in S. enterica taxonomy, diversity and biology.

\section{Results}

142 Salmonella phage genome sequences and patterns of variation

Complete genome sequences of $S$. enterica prophages were searched and downloaded from the NCBI database. Full genome sequences were available for 142 phages (Document S1) and their corresponding genomic information are summarized in Table S1 and include accession number, phage name, assigned cluster, host species, genome size, guanine plus cytosine $(\mathrm{GC})$ content, number of ORFs and virus lineage and DNA structure, i.e., double stranded (dsDNA) or single stranded (ssDNA). The annotated information for the 142 phage genomes was summarized in Document S2. The size range of the phage genomes was from $6.4-\mathrm{kb}$ to $358.7-\mathrm{kb}$, with the majority between $30-\mathrm{kb}$ to $50-\mathrm{kb}$ (Fig. 1a), the GC content ranged from 35.5 to $65.4 \%$ (Table $1 \&$ S1). The virus lineages for all 142 phages were summarized in Table $1 \&$ S1. Ninety-five percent of the phage genomes (135 out of 142) were linear ds DNA and belong to the order Caudovirales and four out of its five known families, namely: Myoviridae, Siphoviridae, Podoviridae and Ackermannviridae based on virus lineages retrieved from Virus-Host DB. There is a total of 27 genera represented in this collection of 142 prophages (Table 1). Four of the remaining seven phages $(5 \%)$ were single stranded DNA (NC_001954.1, NC_006294.1, NC_001332.1 and NC_025824.1), while three have not yet been classified (NC_010393.1, NC_010392.1 and NC_010391.1).

\section{Open reading frame characterization of phage genomes}

The availability of the 142 phage sequences in the NCBI database facilitated comparative genomic analysis. However, 32 out of 142 phages downloaded from the GenBank contained invalid start or stop codons for some ORFs, which were detected during our construction of the Salmonella prophage database (SpDB) and analysis with the Phamerator software (see under Materials and Methods). To ensure congruence between the annotations shown in the GenBank and ORFs displayed by the Pharmerator, it became necessary to ensure that proper start and stop codons were present in the sequences. The detailed error messages (including number of errors and their locations in the original sequences) are shown in Table S1, and the revised sequences and NCBI files are now included in Document S2. The distribution of the genome sizes mirrored the number of ORFs, with the genome size (grey) matching the number of ORFs (blue) as displayed in Fig. 1a and b. For instance, the 4 genomes with the smallest size $(6408,6744$, 7107 and $8454 \mathrm{bp}$ ) had the least ORFs $(10,9,12$, and 10, respectively). Similarly, the 10 largest genomes encoded the highest number of ORFs, typically over 120 ORFs (Table S1). There was a statistically significant, strong linear correlation between the genome sizes and number of ORFs $\left(\mathrm{R}^{2}=0.95, p<0.001\right.$, Fig. 1c).

\section{Salmonella phages occur in other bacteria}

Although the 142 prophages were identified in Salmonella enterica strains present in the Salfos database [17], 




many prophages matched sequences of viral origin associated with bacterial hosts other than Salmonella. This designation of a non-Salmonella host was presumably a consequence of which host the prophage was associated with at the time of initial documentation or publication. The original known host lineage for each phage was used to evaluate the occurrence of these phages in other bacteria. As shown in Table S1 and illustrated in Fig. 2, 
Table 1 The characteristics of 142 prophages present in Salmonella enterica

\begin{tabular}{ll}
\hline Characters & Range or Number \\
\hline Genome size (bp) & From 6408 to 358,663 \\
GC (\%) & From 35.5 to 65.4 \\
Open Reading Frame & From 9 to 545 \\
Clusters & 15 \\
Prophage lineage_Family & 5 \\
Prophage lineage_Genus & 27 \\
Original host lineage_Family & 15 \\
Original host lineage_Genus & 24
\end{tabular}

fifty-three out of the 142 Salmonella phages (37.3\%) were apparently first recovered from the genus Escherichia, followed by 34 phages (23.9\%) first described for a Salmonella host. The others, including Shigella, Burkholderia, and Pseudomonas, showed relatively lower frequencies of 9, 6, and 6 phages, respectively (Fig. 2). Although the cellular host for the phage $\mathrm{P} 4$ is named as Escherichia, it is indeed a satellite virus for another phage called Escherichia virus P2, the latter serving as a helper to provide late gene functions for phage P4 lytic growth cycle, but not for its early functions especially
DNA synthesis and lysogenization [36, 37]. The host of each prophage was detected at a $97 \%$ agreement with the metadata on the bacterial host documented in the Virus-Host Database (Table S1).

\section{Similarities among the 142 phage genomes based on nucleotide identity}

Given that nucleotide identity and genome alignment are key tools for comparative genomic analysis and cluster assignment, NUCmer/MUMmer software was initially applied to analyze these 142 prophage sequences. The pairwise nucleotide identity was calculated among all the 142 genomes and those fragments with over $80 \%$ identity between two genomes were listed in Table S2 The sizes of aligned phage genome fragments varied, ranging from $103 \mathrm{bp}$ to $14,505 \mathrm{bp}$. Out of the 142 genomes investigated, 133 shared at least one fragment with another prophage. We found two phage genomes namely, Salmonella_phage_SJ46 (103 kb) and Enterobacteria_phage_P1 (95 kb), to share an exceptionally large number of fragments with other Salmonella prophages as shown in Fig. 3. In a striking contrast, Salmonella/ Cronobacter prophage vB_CsaM_GAP32 and Salmonella/cyanophage MED4-213, which have the two biggest

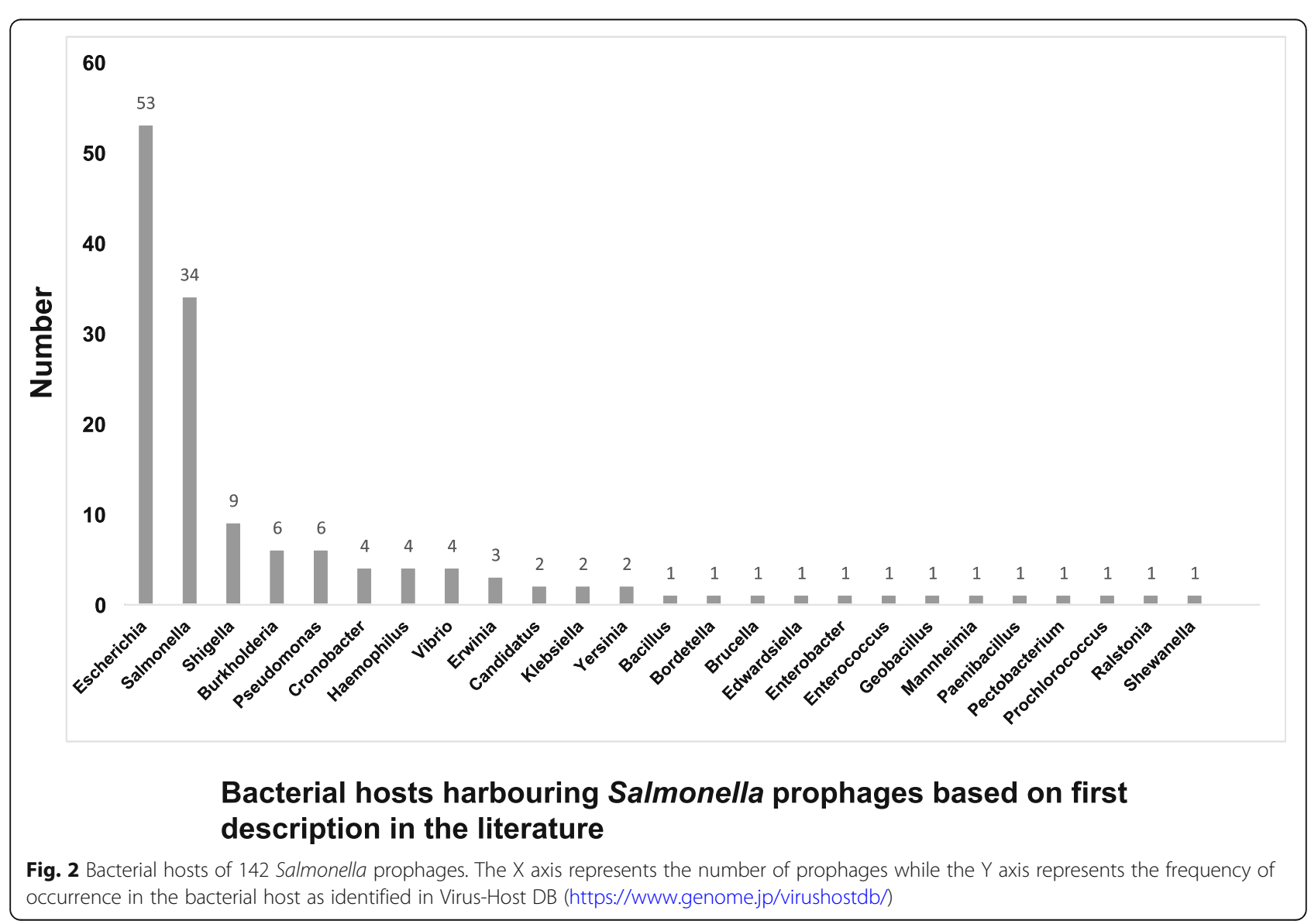


genomes (181- and 359-kb) did not share any fragment with another phage genome.

\section{Clustering of phage genomes}

Conserved DNA fragments among groups of prophage sequences (Fig. 3), were combined with the results of ANI and whole genome dot plot analysis, to assign the prophage genomes to clusters. To this end, a phylogenetic tree from the genome nucleotide identity matrix generated with the Kalign algorithm (Fig. S1). Furthermore, all 142 genomes were concatenated into a single nucleotide sequence and duplicated to form two axes for the purpose of generating a dot plot matrix (Fig. 4). We were able to assign 90 phage genomes into 17 clusters, named A to Q as follows: Cluster A $(n=3)$, Cluster B $(n=5)$, Cluster $\mathrm{C}(n=2)$, Cluster $\mathrm{D}(n=15)$, Cluster $\mathrm{E}$ $(n=4)$, Cluster F $(n=9)$, Cluster $\mathrm{G}(\mathrm{n}=5)$, Cluster $\mathrm{H}$ $(n=10)$, Cluster I $(n=4)$, Cluster J $(n=6)$, Cluster K

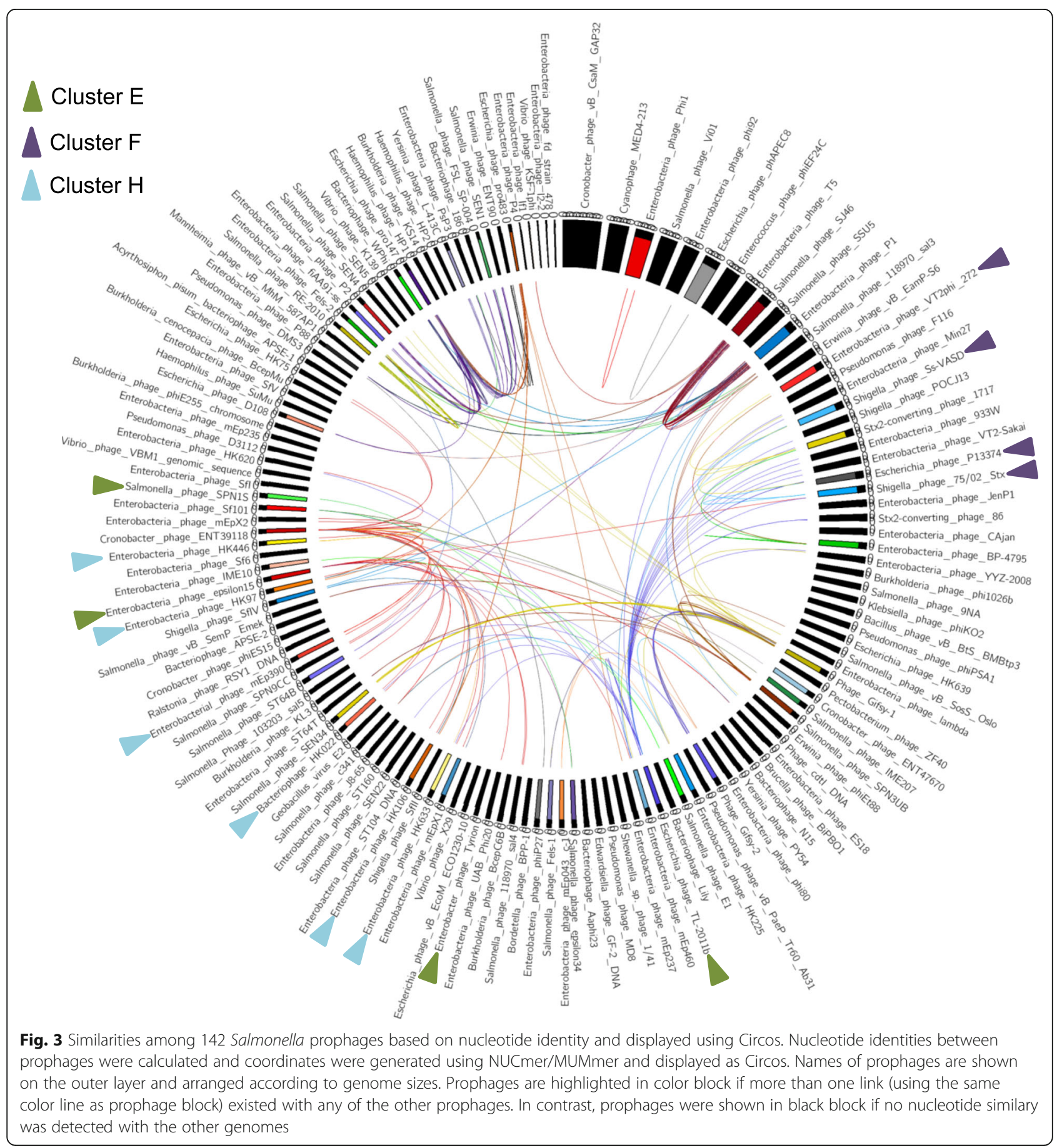


$(n=12)$, Cluster $\mathrm{L}(\mathrm{n}=3)$, Cluster $\mathrm{M}(\mathrm{n}=3)$, Cluster $\mathrm{N}$ $(\mathrm{n}=3)$, Cluster $\mathrm{O}(\mathrm{n}=2)$, Cluster $\mathrm{P}(\mathrm{n}=2)$ and Cluster $\mathrm{Q}(\mathrm{n}=2)$. The remaining 52 phage genomes could not be assigned to any cluster and remained as singletons. We observed both qualitative and quantitative differences in the structure of the clusters based on the intensity of the dot plots (Fig. 4) and pairwise nucleotide similarity between members of each cluster (Table S3,
Cluster A-Q). Clusters E, F, H, I and J had relatively high intracluster nucleotide similarities and moderate genome sizes $(37-77 \mathrm{~kb})$. All four members of Cluster E belonged to the same genus, Epsilon15 virus under the family of Podoviridae according to the International Committee on Taxonomy of Viruses (ICTV) classification. Details of cluster assignment for all prophages are shown in Table S1. We observed uniformity among the

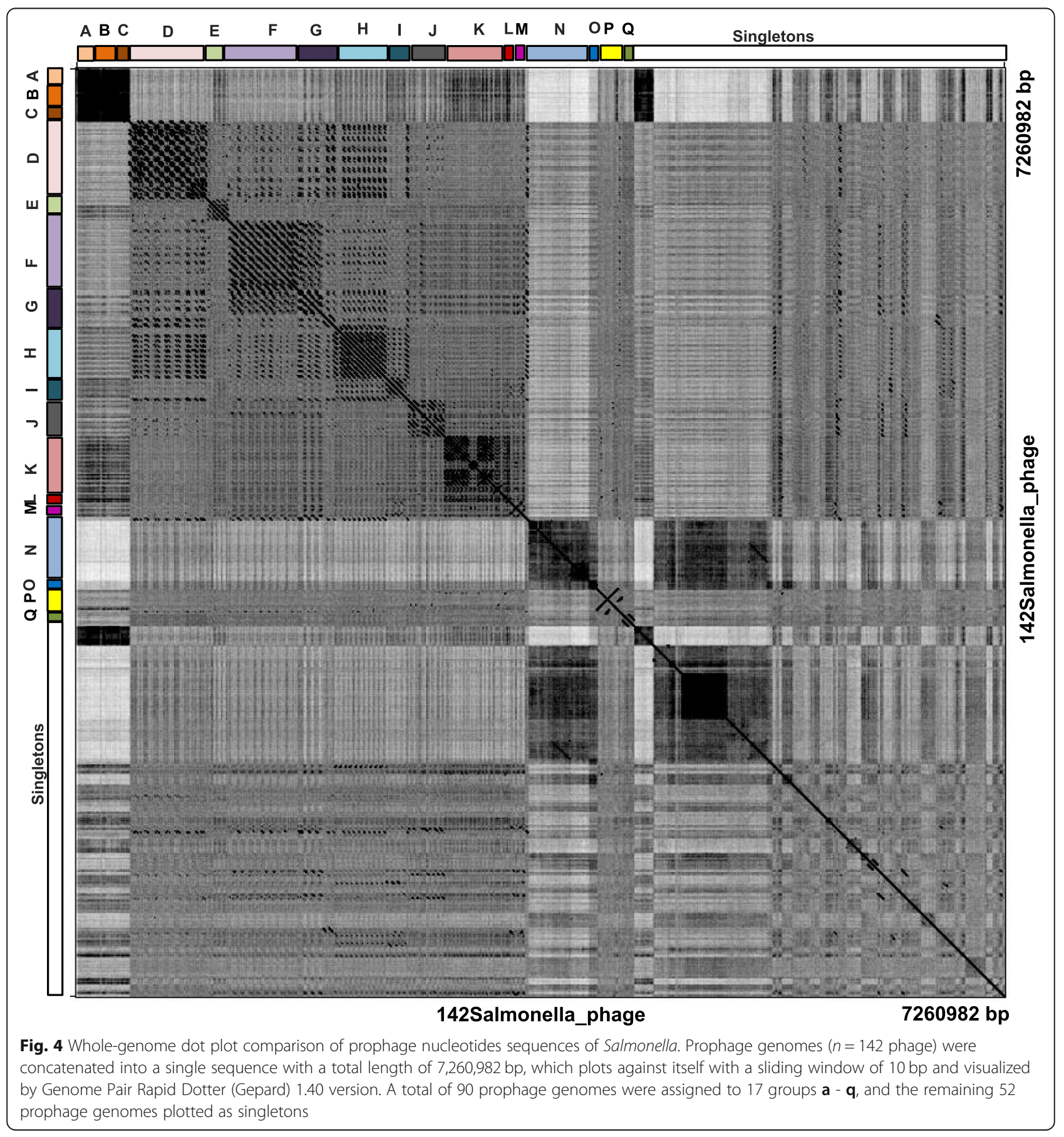


genome sizes and number of ORFs of members of the same cluster (Fig. 1c) which underscores the nucleotide identity among related genomes as also shown in Fig. 3.

\section{Genome maps of multiple phages that incorporate and} display nucleotide and amino acid sequence relationships Using a ClustalW threshold of 35\% amino acid identity and a BLASTP score of 1e-50, the predicted ORFs and translated nucleotide sequences were assigned to groups of closely related sequences using the Phamerator software (Document S3 and Fig. 5). A total of 5796 Phamilies was assigned by Phamerator (Table S4). The most common Phamily was present in 49 prophages but there were 4330 Phamilies found in only one prophage. The relatively conserved Phamily numbers were summarized in the 17 assigned clusters in Table $2 \&$ S5. To establish cluster-specific markers, we retrieved the conserved phamilies from each analyzed clusters and found that a total of 181 representative protein groups were present in all 17 clusters and 159 of them (excluding the 22 bold highlighted proteins in Table S5) were specifically present in one cluster. For example, Cluster A uniquely contained seven Phamilies. In contrast, Cluster $\mathrm{H}$ contained 10 Phamilies but not all were unique because two of these Phamilies were also present in Cluster I. In the same vein, Cluster K contained 15 Phamilies, seven of which were shared with Cluster L. Thus, we demonstrated the presence of unique proteins and/or unique combination of proteins that define each prophage cluster, notwithstanding the fact that some individuals' proteins may be shared among some clusters. A representative genomic map of phages in Cluster $\mathrm{H}$ is shown in Fig. 5. Considerable genome length was observed to be conserved between members of the same cluster inferring synteny (violet shading blocks), with the same phamily ORF (same colour, Fig. 5). Often syntenic regions are interspersed with dissimilar and variable sequences (white blocks or breaks).

\section{Discussion}

We have carried out a comparative genomic analysis for the purpose of characterizing the prophages of Salmonella enterica. Both dsDNA and ssDNA viruses were represented in our collection of 142 phage genomes. The four ssDNA phages present in our collection belonged to the family Inoviridae. In contrast, the dsDNA phages were spread over four of the five known families of the order Caudovirales, i.e., Myoviridae, Podoviridae, Siphoviridae and the rare Ackermannviridae. Within these four families, a total of 27 different phage genera were represented (Table 1). Earlier studies using core genes analysis indicated that Salmonella phages could be classified into five groups, namely: P27-like, P2-like, lambdoid, P22-like, and T7-like [9, 10], and all of which were present in our prophage collection. From our classification, we have identified two new members of Cluster D

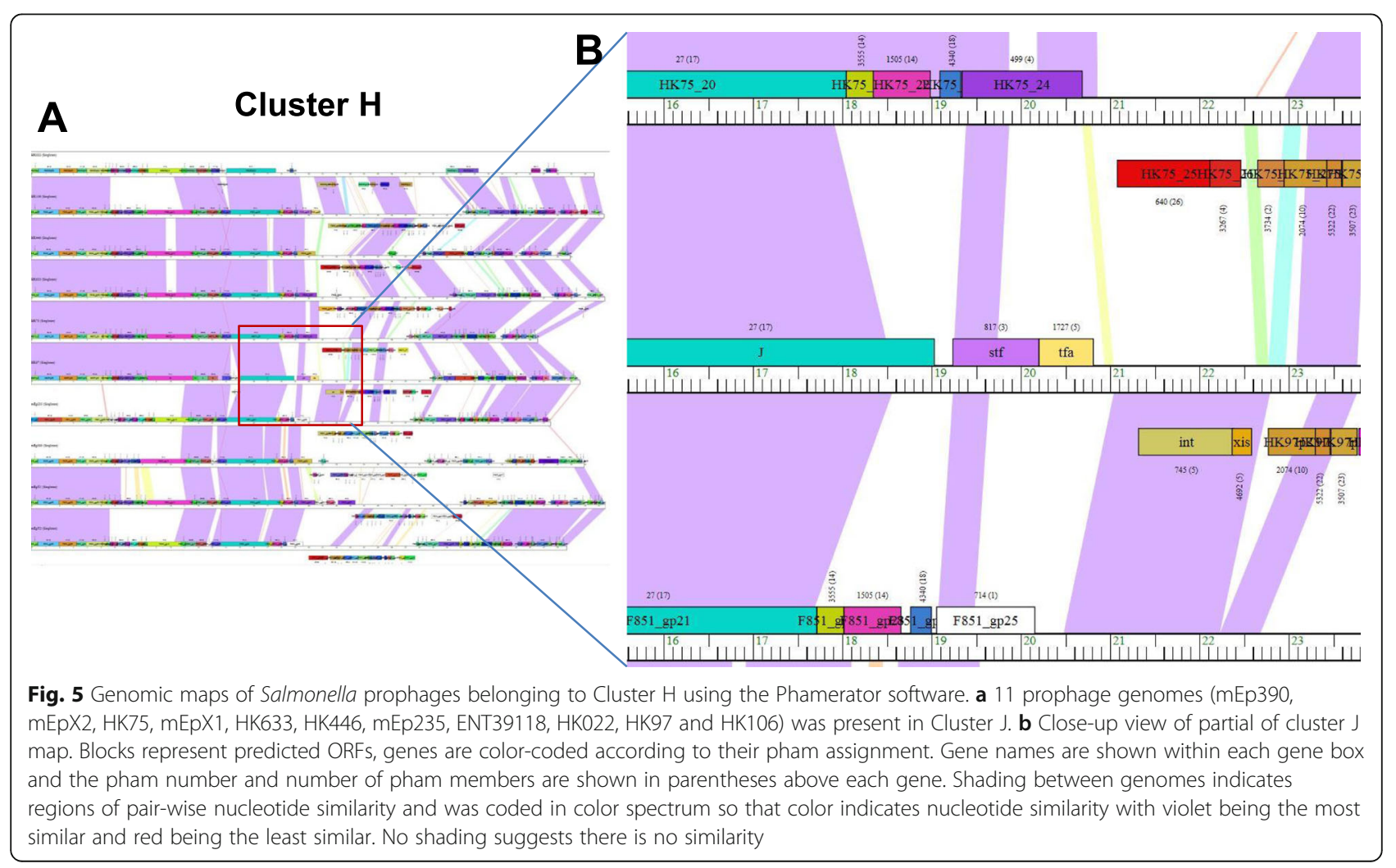


Table 2 The distribution of conserved Phamily members ( $n=$ 181) among clusters of Salmonella bacteriophages

\begin{tabular}{llllll}
\hline Cluster & No. of Pham & Cluster & No. of Pham & Cluster & No. of Pham \\
\hline A & 3 & G & 5 & M & 3 \\
B & 5 & H & 10 & N & 3 \\
C & 2 & I & 4 & O & 2 \\
D & 15 & J & 6 & P & 2 \\
E & 4 & K & 12 & Q & 2 \\
F & 9 & L & 3 & & \\
\hline
\end{tabular}

namely, ST64T and ST104 which are related to the previously described P22-like group. We have described an additional 13 members in this group (Table S1). Similarly, we detected the P2-like PSP3 phages and were able to cluster them with an additional 12 double stranded phage viruses to make up Cluster $\mathrm{K}$. In addition, three lambdoid phages, namely Gifsy 1, Gifsy 2 and lambda were assigned to lambdoid phage group Cluster $M$ (Table S1). This work has extended published observations by identifying additional members of previously described, albeit small groupings, and has achieved a more discriminative and extensive characterization of Salmonella prophage sequences.

An earlier genomic comparison of tailed phages showed 337 fully sequenced lytic and temperate phages in the entire Enterobacteriaceae family [28], and based on this observation, a large number of diverse phages could potentially infect Salmonella. We observed the presence of the same phages infecting different bacteria and whether this is an outcome of the shared location or relatedness among hosts cannot be ascertained at this time. It is possible that both phylogeny, i.e., the relatedness among hosts such as belonging to the same family, or occupation of the same niche, i.e., gastrointestinal tract location may facilitate the presence of same prophages in different hosts. As examples, we observed phages X29 and KSF-1phi in Salmonella, which were first found in Vibrio cholerae according to Virus-Host DB [TAX:666; https://www.genome.jp/virushostdb/]. On the other hand, 38 other phages known to infect Vibrio cholera have not been reportedly found in S. enterica and given that the two organisms belong to different orders, this suggests that hosts phylogeny rather than colocation plays the primary role whether prophages are shared among hosts. Nevertheless, it is difficult to entirely discount the role of a shared niche since the virus will still have to find the new host before infection can take place. Furthermore, 33 phages analyzed here were observed to have originated from Escherichia coli strains [TAX:562] (Table S1). Enterobacteria phage fiAA91-ss is also able to infect at least two more hosts, namely, Shigella sonnei [TAX:624] and Escherichia coli O157:H7 [TAX:83334]. Haemophilus phage Aaphi23 can also infect Aggregatibacter actinomycetemcomitans [TAX: 714] and Haemophilus [TAX:724]. The species A. actinomycetemcomitans has now been renamed Haemophilus actinomycetemcomitans by Potts et al. (1985) [38]. Based on our observations, studies of phage host range should not be restricted to specific species but should comprehensively involve as many different host genera as possible to capture all available information, even if the focus is a particular host species. This will help provide a broader perspective of the distribution of phages and understand how they contribute to the evolution of the host.

The occurrence of the same phage sequences in different hosts may also imply horizontal viral gene transfer among hosts belonging to different genera. Genome clustering facilitates the identification of genes that are in greatest genetic flux and are more likely to have been exchanged horizontally during a relatively recent evolutionary time. Such viral sequence exchanges may help a phage increase its fitness to invade a new host, and evade selective pressure such as anti-phage defense mechanisms [11]. Given the biological arms race between bacteria and phages, and in order to thrive in most environments, phages have evolved multiple tactics to avoid, circumvent or subvert bacterial anti-phage mechanisms [21]. Ironically, these viral sequences once established in Salmonella may help the host to thrive in specific ecological niches, including the gut [39].

Diverse phage genomes were identified in our Salmonella phage collection. As shown in Fig. 2, the highest number of matching prophages were named after the genus Escherichia $(n=53)$ while Salmonella ranked second $(n=34)$. Regarding the lineage for their original known host, three phyla (Firmicutes, Proteobacteria and Cyanobacteria), four classes (Bacilli, Betaproteobacteria, Alphaproteobacteria and Gammaproteobacteria) and 24 unique genera could be identified (Table 1). Such a wide host span provides further evidence of the diversity of Salmonella prophages analyzed in this study. In a study of prophages integrated in a single host species Mycobacterium smegnatis, a threshold of $50 \%$ nucleotide identity was used for genome cluster assignment [24]. The threshold was slightly reduced (45\%) for clustering Pseudomonas phages because phages infecting a genus would be expected to show greater variation in genome sequences than one infecting a single species [23]. Among the 56 phage clusters reported for the Enterobacteriaceae family, the sequence similarity was substantially less between clusters [28], indicating a higher degree of variation and justifying a lower threshold of nucleotide identity for certain clusters in Salmonella phages, a large proportion of which may infect or have previously infected other hosts.

It should be noted that nucleotide identity is not the only parameter for assessing genome properties, because 
the nucleotide alignments for thousands of homologous protein are not significant based on nucleotide alignment, but are clearly homologous based on statistically significant protein structural similarity or strong sequence similarity to an intermediate sequence [40]. Thus, there may not be a linear relationship between sequence identity and function [41]. In our set of phage genomes, except for Cluster B, L and M showed a lower pairwise ANI of $41 \%$, all the other clusters Clusters E (59\%), F (75\%) and J (57\%) displayed high nucleotide identity (Table S3). Their assignment to each of these clusters was supported by results of analysis using the dot plot program, Kalign genome alignment and gene content analysis. For instance, the dotplot (Fig. 4) and Kalign analyses grouped members of Clusters B, G and $\mathrm{N}$, even though some of their respective nucleotide identities were 40.7, 42.2, and 42.3\% (Fig. 4 and Figure S1). A similar phenomenon was also observed for Cluster L made up of members belonging to the same P2 virus group showing a nucleotide identity of $41.3 \%$. The differences in the output of the different tools should not be surprising because of their unique underlying algorithms. While Kalign focuses more on analyzing larger genomes in general, MUMmer focuses more on the similar DNA fragment identification. Despite the high degree of diversity in our prophage collection, we were still able to cluster related isolates using congruent results from at least two bioinformatics analyses.

The genome size ranges of the prophages documented for the different bacteria genera are fairly similar: Salmonella (6.4-358.7 kb), Pseudomonas (3.0$316.0 \mathrm{~kb})$, Staphylococcus (15.6-138.7 kb), Gordonia (17.1-103.4 kb), Bacillus (14.3-497.5 kb) and Mycobacterium (41.9-164.6 kb). The ranges of the GC content showed less of an overlap: Salmonella (35.5 to 65.4\%), Pseudomonas (37.0 to 66.0\%), Staphylococcus (29.3 to 38.0\%), Gordonia (47.0 to 68.8\%), Bacillus (29.9 to $49.9 \%$ ) and Mycobacterium (56.3 to 69.1\%) [23-27]. Salmonella and Pseudomonas both belong to the Enterobacteriaceae family and their phages share very similar genome sizes and GC content. Despite the similarities between the phages of Pseudomonas and Salmonella, the former appears to display better clustering pattern (fewer singletons) based on the grouping of 100 out of 130 phages [23] compared to 90 out of 142 Salmonella phages with 52 singletons. However, as Pseudomonas bacteriophages were collected only using "Pseudomonas" as host for the search in the database [23], the set most likely did not represent the full complement of viruses capable of infecting Pseudomonas and integrating into the genome and would have excluded bacteriophages of this group but first found or described in another bacterial host. We expect that more diverse prophage patterns would be obtained for Pseudomonas and other bacterial hosts if a more comprehensive search of bacterial genomes is carried out with tool such as PHASTER [34].

The diversity of Salmonella prophage genomes was also reflected in the total number of phamilies for the ORFs in the analyzed prophage genomes: 5796. One phamily with a Pham number of 2217 was observed to be dominant and was present in 49 prophages (34.5\% of 142 phages) whereas 4330 phamilies were each present in a single prophage, which makes it challenging to select conserved genes for all the 142 prophage genomes. Clustering of the viral genome was useful in establishing relatedness of Salmonella bacteriophages. In each assigned cluster, some conserved Pham numbers (containing different ORFs) are present. For example, Pham 180 (portal protein), Pham 2012 (recombination protein) and Pham 2217 (endopeptidase) are commonly present in Cluster D; Pham 321 (phage head-tail connector protein), Pham 415 (terminase large subunit) and Pham 1522 (terminase small unit) in Cluster E; Pham 1995 (lysozyme), Pham 2370 (terminator) and Pham 1332 (attachment invasion locus protein precursor) in Cluster F; Pham 27 (phage tail protein), Pham 519 (phage portal protein), and Pham 1717 (assembly protein) in Cluster H; Pham 528 (major capsid protein), Pham 297 (terminase large subunit) and Pham 666 (tail protein) in Cluster J; Pham 963 (base plate assembly protein) in Cluster K (Document S3). Specifically, some proteins are unique to one cluster, for example, four members of Pham 4878 (a hypothetical protein), Pham 1893 (a hypothetical protein) Pham 2968 (a hypothetical protein) Pham 2849 (a hypothetical protein) in the Cluster E. These may be good markers for characterizing prophage members of the different clusters (Document S3, Table S5).

The observations reported in this study are quite relevant for the application of bacteriophages as antibacterial agents and in cloning vector construction. Our list of Salmonella bacteriophages can be used for screening a novel, candidate bacteriophage identified as a potential anti-bacterial agent for Salmonella or any host described in this study. The implication is that because the bacteriophages present in our collection induce lysogeny, the bacterial host will be immune to infection or lysis by the same bacteriophage; a bacteriophage on our list will likely not be an effective antibacterial agent for the hosts identified in this study. Thus, a distinct bacteriophage may be a better anti-bacterial candidate than one on our list. Similarly, the Salmonella prophage database in the Pharmerator can be used to evaluate a candidate antibacterial agent even if it is distinct from members on our list. Because bacteriophages are prone to recombination leading to a mosaic profile, the protein components can be used to assess relatedness with the goal of 
choosing a candidate antibacterial agent that is phylogenetically distant from any of the isolates in our collection to increase the chance of success. In the same vein, knowledge from our collection can be used in strategies to design phage vectors. For example, $\lambda$ cloning vectors require a lytic cycle and their ability to package large foreign DNA fragments have relied on the removal of lysogenic genes from the vectors. Thus, the removal of lysogenic fragments in a temperate phage can probably deviate the life cycle into a lytic path making them more relevant for vector construction especially if the bacteriophage has signature genetic markers that can be exploited for selection or vector purification, e.g., antibacterial resistance genes or a target for a widely used ligand.

\section{Conclusions}

The comparative genomic analysis of 142 Salmonella enterica subsp. enterica prophages revealed a high diversity in genomic characteristics, compared to that in other bacteria species such as Pseudomonas, Staphylococcus, Gordonia, Bacillus and Mycobacterium. The combination of nucleotide identity, dot plot, genome map comparison and gene content analysis, revealed the presence of 17 main clusters of Salmonella phages and many singletons. In order to have a fuller picture of Salmonella phages, a similar comparative phage genomic analysis needs to be performed on Salmonella virulent/ lytic phages. The high diversity among prophages may well be a mechanism developed to generate new molecules and decoys to thwart the potent, anti-viral defence mechanism of the bacterial host. We hypothesize that in place of the resources needed to lyse a host cell, temperate prophages may instead have developed a rather sophisticated capacity to acquire and display diversity and thereby present a degree of invincibility against the host arsenal so that they can survive long enough to integrate into the host genome. Thus, we predict that prophages will show more diversity than their virulent phage counterparts. Areas of conservation and variations among the investigated prophage genomes provides further evidence showing why prophage typing is a discriminative method for Salmonella typing. A fuller understanding of the genomic architecture of Salmonella bacteriophages should furnish practical information relevant for bacterial strain construction, vector development, and the selection of appropriate phages to be tested for bio-control strategies.

\section{Materials and methods}

\section{Phage genome sequences}

We previously identified 154 different prophages among 1760 S. enterica genomes derived from 151 serovars using PHASTER [18]. We downloaded 142 of these 154 phage genomes from NCBI Batch Entrez (https://www. ncbi.nlm.nih.gov/sites/batchentrez) but were unable to locate the full length of the remaining 12 genomes. Genome annotations were downloaded from NCBI and validated using gene calling programs GeneMarkS and Glimmer [42-44], and BLASTN when necessary. The virus lineages information for the 142 prophage genomes, and the original known host lineage for each prophage were both retrieved from the Virus-Host DB (https://www.genome.jp/virushostdb/). In addition, the host of each prophage were further evaluated by using a web based tool called HostPhinder (https://cge.cbs.dtu. $\mathrm{dk} /$ services/HostPhinder/) [45].

\section{Comparative phage genome analysis}

All 142 phage genome sequences were pooled and saved as a multi-fasta file and aligned to one another using MUMmer v4.0.0.beta2. Genome comparisons were carried out to produce delta files using the following breaklen parameters: maxgap $=200$; mincluster $=90$; minmatch $=$ 60. Results were generated as coordinate files using "shwon-coords" and visualized via Circos [46]. To illustrate the nucleotide similiarities among all the analyzed phage genomes, the visualization tool Circos [46] that display connecting lines between prophages was used. Whole genome alignment and calculation of percentage of ANI identity were carried out with Kalign [30]. Evolutionary analyses for tree construction were conducted in MEGA $X$ [47]. Briefly, the evolutionary history was inferred using the Neighbor-Joining method [48]. The bootstrap consensus tree inferred from 500 replicates [49] was taken to represent the evolutionary history of the taxa analyzed. Branches corresponding to partitions reproduced in less than $50 \%$ of bootstrap replicates were collapsed. The percentage of replicate trees in which the associated taxa clustered together in the bootstrap test $(500$ replicates) was shown next to the branches. The evolutionary distances were computed using the Maximum Composite Likelihood method [50] and are presented as the number of base substitutions per site. There were a total of 431,295 positions in the final dataset. Prophage genomes $(n=142$ phage) were concatenated into a single sequence with a total length of 7,260,982 bp, which when plotted against itself with a sliding window of $10 \mathrm{bp}$ and visualized by Gepard 1.40 version [29], revealed an overall pattern of similarity or dissimilarity of all the genomes. The graphics displayed pairwise similarity between genomes which was then used for the preliminary assignment of clusters. Among all the analyzed prophage genomes, if two sequences shared high similarity, a diagonal would show at that location on the plot (the center diagonal line demonstrated the 
$100 \%$ similarities where a sequences was compared to itself).

\section{Genome clustering}

Three criteria were used to cluster the phage genomes. First, the genomes were grouped based on nucleotide identity among members. Second, dot plot was used to analyze sequences based on similarity leading to graphically demonstrable clustering of sequences. Third, translated nucleotide sequences were used to cluster phages based on translated amino acids sequences. Phage genomes that did not meet these criteria were identified as 'singletons'.

\section{Salmonella phage database creation and genome map viewing via Phamerator}

In order to produce the first, web-based inventory of Salmonella prophages that could be used for comparative analysis with prophage genomes from other bacteria, we created the SpDB in the Pharmerator platform. For this purpose, Salmonella phage database, a web-based application PhamDB was used for building the Salmonella Phamerator phage database consisting of 142 phage sequences. Briefly, after installing Docker Toolbox, Kitematic was launched to finish the initial setup and loading. An existing 'PhamDB' database in the Phamerator platform was downloaded and used as a template. By running the PhamDB program as a web interface on a local network, a new database was created in toolbar using GenBank Files as inputs. All the 142 phage NCBI files were summarized in Document S2. The generated database was a sql file which was used as an input file and uploaded into Phamerator website (https://phamerator.org, created and maintained by Dr. Steven Cresawn of James Madison University). Based on the assigned clusters, genome maps can be visualized for direct comparisons. As displayed in the Phamerator map, long regions of violet shading indicate long conserved regions between phage genomes. Within a cluster, the same color block represents the ORF with higher similarities. Regions of high similarity and same-coloured ORF blocks shown on the map indicated a prevalent synteny. Areas with little or no sequence similarity between genome sequences are shown as either white blocks or a break in a syntenic block.

\section{Supplementary information}

Supplementary information accompanies this paper at https://doi.org/10. 1186/s12864-020-6765-z.

Additional file 1: Figure S1. Phylogenetic tree of 142 Salmonella prophages based on genome alignment and nucleotide identity using Kalign. The relationships among the Salmonella isolates based on genome alignments and \% nucleotide identity were inferred using the Neighbor-Joining method and conducted in MEGA X.
Additional file 2: Table S1. Detailed profiles of 142 prophages present in Salmonella enterica.

Additional file 3: Table S2. Nucleotide similarities between pairs of Salmonella prophage genomes $(n=142)$.

Additional file 4: Table S3. Nucleotide identify matrix for 17 clusters of Cluster A-Q

Additional file 5: Table S4. All the assigned Pham numbers and its color code.

Additional file 6: Table S5. The distribution of conserved Phamily members among clusters of Salmonella bacteriophages.

Additional file 7: Document S1. Multi-fasta files for the 142 Salmonella prophage sequences.

Additional file 8: Document S2. NCBI files of 142 prophages used for Phamerator database creation.

Additional file 9: Document S3. Scalable Vector Graphics (SVG) figure demonstration files for the 17 assigned clusters of 142 prophages.

\section{Abbreviations}

ORFs: Open Reading Frames; PHASTER: PHAge Search Tool Enhanced Release; CRISPRs: clustered regularly interspaced short palindromic repeats; ICTV: International Committee on Taxonomy of Viruses; Gepard: Genome Pair Rapid Dotter; dsDNA: Double stranded DNA; ssDNA: Single stranded DNA

\section{Acknowledgements}

We thank Dr. Walid Mottawea of the University of Ottawa, Canada for assistance in prophage sequence analysis, and Dr. Steven Cresawn from James Madison University for assistance with uploading the Salmonella phage Database on the Phamerator website (https://phamerator.org). We acknowledge excellent review comments by Ray Theoret, Hongsheng Huang and Amit Matthews.

\section{Authors' contributions}

RG and DO conceived and designed the study. RG developed and analyzed the data and wrote the draft manuscript. SN, SM, RL analyzed data and edited manuscript. LG edited manuscript. DO supervised the project, analyzed data and edited manuscript. All authors read and approved the final manuscript.

\section{Funding}

RG was supported by the Genome Canada funded project titled "A SystOMICS approach to ensuring food safety and reducing the economic burden of Salmonellosis". DO's research program has received funding support from Genome Research and Development Initiative of the Government of Canada, Ontario Ministry of Agriculture, Food and Rural Affairs, Canadian Security and Science Program of the Department of National Defence and the Canadian Food Inspection Agency. SM holds a Tier 1 Canada Research Chair in Bacteriophages. LG is the Leung Family Professor of Food Safety in University of Guelph, Guelph.

Availability of data and materials

The datasets supporting the conclusions of this article are included within the article and its additional files.

Ethics approval and consent to participate

Not applicable.

Consent for publication

This manuscript was approved for publication by the Canadian Food Inspection Agency.

Competing interests

The authors declare that they have no competing interests.

\section{Author details}

'Ottawa Laboratory Fallowfield, Canadian Food Inspection Agency, Ottawa, Ontario, Canada. ${ }^{2}$ Department of Food Science and Agricultural Chemistry, McGill University, Ste Anne de Bellevue, QC, Canada. ${ }^{3}$ Félix d'Hérelle Reference Center for Bacterial Viruses, Faculté de médecine dentaire, 
Université Laval, Québec City, QC GIV OA6, Canada. ${ }^{4}$ Groupe de recherche en écologie buccale, Faculté de médecine dentaire, Université Laval, Québec City, QC GIV OA6, Canada. ${ }^{5}$ Département de biochimie, de microbiologie, et de bio-informatique, Faculté des sciences et de génie, Université Laval, Québec City, QC GIV 0A6, Canada. Institut de Biologie Intégrative et des Systèmes, Université Laval, Québec City, QC G1V 0A6, Canada. 'Present Address:Department of Food Science, University of Guelph, Guelph, Ontario, Canada.

Received: 2 October 2019 Accepted: 30 April 2020

Published online: 26 May 2020

\section{References}

1. Fabrega A, Vila J. Salmonella enterica serovar Typhimurium skills to succeed in the host: virulence and regulation. Clin Microbiol Rev. 2013;26(2):308-41

2. Su LH, Chiu CH. Salmonella: clinical importance and evolution of nomenclature. Chang Gung Med J. 2007;30(3):210-9.

3. Gal-Mor O, Boyle EC, Grassl GA. Same species, different diseases: how and why typhoidal and non-typhoidal Salmonella enterica serovars differ. Front Microbiol. 2014;5:391.

4. Jantsch J, Chikkaballi D, Hensel M. Cellular aspects of immunity to intracellular Salmonella enterica. Immunol Rev. 2011;240(1):185-95.

5. Hohmann EL. Nontyphoidal salmonellosis. Clin Infect Dis. 2001;32(2):263-9.

6. Ray CG, Ryan KJ, editors. Sherris medical microbiology. 4th ed. New York: McGraw-Hill; 2004..

7. Lin DM, Koskella B, Lin HC. Phage therapy: an alternative to antibiotics in the age of multi-drug resistance. World J Gastrointest Pharmacol Ther. 2017; 8(3):162-73.

8. Wommack KE, Colwell RR. Virioplankton: viruses in aquatic ecosystems Microbiol Mol Biol Rev. 2000;64(1):69-114.

9. Kropinski AM, Sulakvelidze A, Konczy P, Poppe C. Salmonella phages and prophages--genomics and practical aspects. Methods Mol Biol. 2007;394: 133-75.

10. Switt Al, Sulakvelidze A, Wiedmann M, Kropinski AM, Wishart DS, Poppe C, et al. Salmonella phages and prophages: genomics, taxonomy, and applied aspects. Methods Mol Biol. 2015;1225:237-87.

11. Labrie SJ, Samson JE, Moineau S. Bacteriophage resistance mechanisms. Nat Rev Microbiol. 2010;8(5):317-27.

12. Doron S, Melamed S, Ofir G, Leavitt A, Lopatina A, Keren M, et al. Systematic discovery of antiphage defense systems in the microbial pangenome. Science. 2018;359(6329):eaar4120.

13. Levesque S, Moineau S. A stockpile of antiviral defences. Nature. 2018; 556(7701):318-9.

14. Ackermann HW. Tailed bacteriophages: the order caudovirales. Adv Virus Res. 1998:51:135-201.

15. Adriaenssens EM. Analysis of spounaviruses as a case study for the overdue reclassification of tailed phages. Syst Biol. 2020;69(1):110-23.

16. Duckworth DH. Who discovered bacteriophage? Bacteriol Rev. 1976;40(4): 793-802.

17. Boyd JS. The symbiotic bacteriophages of Salmonella typhimurium. J Pathol Bacteriol. 1950;62(4):501-17.

18. Mottawea W, Duceppe MO, Dupras AA, Usongo V, Jeukens J, Freschi L, et al. Salmonella enterica prophage sequence profiles reflect genome diversity and can be used for high discrimination subtyping. Front Microbiol. 2018;9: 836.

19. Brussow H, Canchaya C, Hardt WD. Phages and the evolution of bacterial pathogens: from genomic rearrangements to lysogenic conversion. Microbiol Mol Biol Rev. 2004;68(3):560-602 table of contents.

20. Colavecchio A, D'Souza Y, Tompkins E, Jeukens J, Freschi L, Emond-Rheault $\mathrm{JG}$, et al. Prophage integrase typing is a useful indicator of genomic diversity in Salmonella enterica. Front Microbiol. 2017;8:1283.

21. Nicolle P, Vieu JF, Diverneau G. Supplementary lysotyping of vi-positive strains of Salmonella typhi, insensitive to all the adapted preparations of Craigie's vi II phage (group I+IV). Arch Roum Pathol Exp Microbiol. 1970; 29(4):609-17.

22. Anderson ES, Ward LR, Saxe MJ, de Sa JD. Bacteriophage-typing designations of Salmonella typhimurium. J Hyg (Lond). 1977;78(2):297-300.

23. Ha AD, Denver DR. Comparative genomic analysis of 130 bacteriophages infecting bacteria in the genus Pseudomonas. Front Microbiol. 2018;9:1456.
24. Hatfull GF, Jacobs-Sera D, Lawrence JG, Pope WH, Russell DA, Ko CC, et al. Comparative genomic analysis of 60 Mycobacteriophage genomes: genome clustering, gene acquisition, and gene size. J Mol Biol. 2010;397(1):119-43.

25. Kwan T, Liu J, DuBow M, Gros P, Pelletier J. The complete genomes and proteomes of 27 Staphylococcus aureus bacteriophages. Proc Natl Acad Sci U S A. 2005;102(14):5174-9.

26. Grose JH, Jensen GL, Burnett SH, Breakwell DP. Genomic comparison of 93 Bacillus phages reveals 12 clusters, 14 singletons and remarkable diversity. BMC Genomics. 2014;15:855.

27. Pope WH, Mavrich TN, Garlena RA, Guerrero-Bustamante CA, Jacobs-Sera D, Montgomery MT, et al. Bacteriophages of Gordonia spp. Display a spectrum of diversity and genetic relationships. MBio. 2017;8(4):e01069-17.

28. Grose $\mathrm{JH}$, Casjens SR. Understanding the enormous diversity of bacteriophages: the tailed phages that infect the bacterial family Enterobacteriaceae. Virology. 2014;468-470:421-43.

29. Krumsiek J, Arnold R, Rattei T. Gepard: a rapid and sensitive tool for creating dotplots on genome scale. Bioinformatics. 2007;23(8):1026-8.

30. Lassmann T, Sonnhammer EL. Kalign--an accurate and fast multiple sequence alignment algorithm. BMC Bioinformatics. 2005;6:298.

31. Kurtz S, Phillippy A, Delcher AL, Smoot M, Shumway M, Antonescu C, et al. Versatile and open software for comparing large genomes. Genome Biol. 2004;5(2):R12.

32. Cresawn SG, Bogel M, Day N, Jacobs-Sera D, Hendrix RW, Hatfull GF. Phamerator: a bioinformatic tool for comparative bacteriophage genomics. BMC Bioinformatics. 2011;12:395.

33. Lamine JG, DeJong RJ, Nelesen SM. PhamDB: a web-based application for building Phamerator databases. Bioinformatics. 2016;32(13):2026-8.

34. Arndt D, Grant JR, Marcu A, Sajed T, Pon A, Liang Y, et al. PHASTER: a better, faster version of the PHAST phage search tool. Nucleic Acids Res. 2016; 44(W1):W16-21.

35. Zhou Y, Liang Y, Lynch KH, Dennis JJ, Wishart DS. PHAST: a fast phage search tool. Nucleic Acids Res. 2011;39(Web Server issue):W347-52.

36. Haggard-Ljungquist E, Jacobsen E, Rishovd S, Six EW, Nilssen O, Sunshine MG, et al. Bacteriophage P2: genes involved in baseplate assembly. Virology. 1995;213(1):109-21.

37. Six EW. The helper dependence of satellite bacteriophage P4: which gene functions of bacteriophage P2 are needed by P4? Virology. 1975;67(1):249-63.

38. Potts TV, Zambon JJ, Genco RJ. Reassignment of Actinobacillus actinomycetemcornitans to the genus Haemophilus as Haemophilus actinornycetemcomitans comb. nov. Int J Syst Bacteriol. 1985;35(3):337-41.

39. Shkoporov AN, Hill C. Bacteriophages of the human gut: the "known unknown" of the microbiome. Cell Host Microbe. 2019:25(2):195-209.

40. Pearson WR. An introduction to sequence similarity ("homology") searching. Curr Protoc Bioinformatics. 2013;Chapter 3:Unit3 1.

41. Joshi T, Xu D. Quantitative assessment of relationship between sequence similarity and function similarity. BMC Genomics. 2007:8:222.

42. Besemer J, Borodovsky M. Heuristic approach to deriving models for gene finding. Nucleic Acids Res. 1999;27(19):3911-20.

43. Delcher AL, Harmon D, Kasif S, White O, Salzberg SL. Improved microbial gene identification with GLIMMER. Nucleic Acids Res. 1999;27(23):4636-41.

44. Mills R, Rozanov M, Lomsadze A, Tatusova T, Borodovsky M. Improving gene annotation of complete viral genomes. Nucleic Acids Res. 2003;31(23):7041-55.

45. Villarroel J, Kleinheinz KA, Jurtz VI, Zschach H, Lund O, Nielsen M, et al. HostPhinder: A Phage Host Prediction Tool. Viruses. 2016;8(5):116.

46. Krzywinski M, Schein J, Birol I, Connors J, Gascoyne R, Horsman D, et al. Circos: an information aesthetic for comparative genomics. Genome Res. 2009:19(9):1639-45.

47. Kumar S, Stecher G, Li M, Knyaz C, Tamura K. MEGA X: molecular evolutionary genetics analysis across computing platforms. Mol Biol Evol. 2018;35(6):1547-9.

48. Saitou N, Nei M. The neighbor-joining method: a new method for reconstructing phylogenetic trees. Mol Biol Evol. 1987;4(4):406-25.

49. Felsenstein J. Confidence limits on phylogenies: an approach using the bootstrap. Evolution. 1985:39(4):783-91.

50. Tamura K, Nei M, Kumar S. Prospects for inferring very large phylogenies by using the neighbor-joining method. Proc Natl Acad Sci U S A. 2004;101(30): 11030-5.

\section{Publisher's Note}

Springer Nature remains neutral with regard to jurisdictional claims in published maps and institutional affiliations. 